\title{
SOME MATHEMATICAL ANALYSIS IN SEMICONDUCTOR DEVICES
}

\author{
L. HSIAO*
}

\begin{abstract}
This is a survey on the progress obtained recently in understanding of the relation between the hydrodynamic models and drift-diffusion models in semiconductor devices. The relationship is investigated by two kinds of efforts: to study the relaxation limit problems from the hydrodynamic models to the drift-diffusion models, and to compare the large-time behavior of these models. The first concerns to weak entropy solutions for the hydrodynamic models, while the second is for smooth solutions. Both Cauchy problem and Initial-boundary value problems are studied.
\end{abstract}

1. Introduction. The theory of semiconductor devices modeling and its mathematical analysis have attracted a lot of attention in both applied mathematics and semiconductor physics. To remedy the high cost in dealing with the basic kinetic transport equations in real applications, one may derive some simpler fluid dynamical equations for macroscopic quantities, such as particle density, current density or energy density and gives a good compromise between the physical accuracy and the reduction of computational cost. The hydrodynamic model and drift-diffusion model, which are two kinds of important mathematical models in semiconductor devices, were introduced by this consideration. The hydrodynamic model plays an important role in simulating the behavior of the charge carrier in submicron semiconductor devices since it exhibits velocity overshoot and ballistic effects which are not accounted for in the classical drift-diffusion model. The detailed introduction to these two kinds of models can be found in the books by Jerome [10], by Markowich, Ringhofer and Schmeiser [17], etc.

There has been a lot of work to understand the relation between these two kinds of models recently. The aim of this paper is to show the progress obtained in understanding the relation in both unipolar case and bipolar case.

The hydrodynamic model in the case of one carrier type(e.g. electrons), called unipolar case also, can be written in one space dimension as

$$
\left\{\begin{array}{l}
\rho_{t}+(\rho u)_{x}=0 \\
(\rho u)_{t}+\left(\rho u^{2}+p(\rho)\right)_{x}=\rho \Phi_{x}-\frac{\rho u}{\tau} \\
\Phi_{x x}=\rho-D(x)
\end{array}\right.
$$

where $\rho, u, \Phi$ denote the electron density, velocity, and electrostatic potential respectively. The pressure function $p=p(\rho)$ has the property that $\rho^{2} p^{\prime}(\rho)$ is strictly increasing function from $[0, \infty)$ onto $[0, \infty)$. A commonly-used hypothesis is

$$
p(\rho)=\frac{\rho^{\gamma}}{\gamma}, \quad \gamma>1
$$

$\tau>0$ is the momentum relaxation time, the doping profile $D=D(x)$ is bounded.

By introducing the current density $m=\rho u,(1.1)$ becomes

$$
\left\{\begin{array}{l}
\rho_{t}+m_{x}=0 \\
m_{t}+\left(\frac{m^{2}}{\rho}+p(\rho)\right)_{x}=\rho \Phi_{x}-\frac{m}{\tau} \\
\Phi_{x x}=\rho-D(x)
\end{array}\right.
$$

${ }^{*}$ Academy of Mathematics and Systems Sciences, Chinese Academy of Sciences, Beijing 100080, China. 
The bipolar hydrodynamic model takes the form

$$
\left\{\begin{array}{l}
\alpha_{t}+m_{x}=0 \\
m_{t}+\left(\frac{m^{2}}{\alpha}+p(\alpha)\right)_{x}=\alpha \Phi_{x}-\frac{m}{\tau} \\
\beta_{t}+n_{x}=0 \\
n_{t}+\left(\frac{n^{2}}{\beta}+q(\beta)\right)_{x}=-\beta \Phi_{x}-\frac{n}{\tau} \\
\Phi_{x x}=\alpha-\beta-D(x)
\end{array}\right.
$$

where $\alpha, \beta, m, n$ and $\Phi$ denote the electron density, the positively charged hole density, the electron and the hole current densities, and the electrostatic potential respectively. The pressure function $p(\alpha)$ and $q(\beta)$ take the forms as

$$
\begin{array}{ll}
p(\alpha)=\frac{\alpha^{\gamma_{\alpha}}}{\gamma_{\alpha}}, & \gamma_{\alpha}>1 \\
q(\beta)=\frac{\beta^{\gamma_{\beta}}}{\gamma_{\beta}}, & \gamma_{\beta}>1
\end{array}
$$

The current relaxation time $\tau$ is a positive constant, the doping profile $D=D(x)$ is bounded.

The drift-diffusion model corresponding to the unipolar case has the form

$$
\left\{\begin{array}{l}
\rho_{t}+\left[\rho \Phi_{x}-p(\rho)_{x}\right]_{x}=0 \\
\Phi_{x x}=\rho-D(x) \\
m=\rho \Phi_{x}-p(\rho)_{x}
\end{array}\right.
$$

And the corresponding form to the bipolar case is

$$
\left\{\begin{array}{l}
\alpha_{t}+\left[\alpha \Phi_{x}-p(\alpha)_{x}\right]_{x}=0 \\
\beta_{t}-\left[\beta \Phi_{x}+q(\beta)_{x}\right]_{x}=0 \\
\Phi_{x x}=\alpha-\beta-D(x) \\
m=\alpha \Phi_{x}-p(\alpha)_{x} \\
n=-\beta \Phi_{x}-q(\beta)_{x}
\end{array}\right.
$$

The relationship of the models (1.1) and (1.3) or the models (1.2) and (1.4) has been investigated by two kinds of efforts, namely, to study the relaxation limit problem or to compare the large-time behavior of these models. To explain the first kind of efforts, let us consider the system (1.1). Introducing the new time scale $s=\tau t$ and denoting the corresponding scaled quantities by $\rho^{\tau}(x, s)=\rho\left(x, \frac{s}{\tau}\right), m^{\tau}(x, s)=$ $\frac{1}{\tau} m\left(x, \frac{s}{\tau}\right), \Phi^{\tau}(x, s)=\Phi\left(x, \frac{s}{\tau}\right)$, we get the system on $\left(\rho^{\tau}, m^{\tau} . \Phi^{\tau}\right)$

$$
\left\{\begin{array}{l}
\rho_{s}^{\tau}+m_{x}^{\tau}=0 \\
\tau^{2} m_{s}^{\tau}+\tau^{2}\left(\frac{\left(m^{\tau}\right)^{2}}{\rho^{\tau}}\right)_{x}+p\left(\rho^{\tau}\right)_{x}=\rho^{\tau} \Phi_{x}^{\tau}-m^{\tau} \\
\Phi_{x x}^{\tau}=\rho^{\tau}-D(x)
\end{array}\right.
$$

The strong limits of $\rho^{\tau}, m^{\tau}$ and $\Phi^{\tau}$ (if they exist), denoted by $\tilde{\rho}, \tilde{m}, \tilde{\Phi}$, would satisfy

$$
\left\{\begin{array}{l}
\tilde{\rho}_{s}+\tilde{m}_{x}=0 \\
\tilde{m}=\tilde{\rho} \tilde{\Phi}_{x}-p(\tilde{\rho})_{x} \\
\tilde{\Phi}_{x x}=\tilde{\rho}-D(x)
\end{array}\right.
$$


This gives the system (1.3).

There are results to prove this convergence as $\tau \rightarrow 0$ on weak entropy solutions to (1.1) or (1.2) which will be introduced in section 2. It is clear that the zero relaxation limit problem is closely connected to the asymptotic behavior of solutions. The section 3 is devoted to this effort to show that these two systems (1.1) and (1.3) (or (1.2) and (1.4)) have the same time asymptotic behavior to the corresponding smooth solutions. This paper is concentrated only on the relationship of the hydrodynamic models and drift diffusion models. There are many other results related to these models such as [1] [2] [5] [11] [12] [16], etc. Although both Cauchy problem and initial boundary value problem will be discussed in this paper, the emphasis will be on initial-boundary value problem.

2. Zero relaxation limit problem. The global existence of weak solutions of the Cauchy problem to the system (1.1) was established by Marcati and Natalini in [14] for the case $1<\gamma \leq \frac{3}{5}$. Furthermore they studied the zero relaxation limit of the hydrodymic model towards the drift-diffusion model in [15] for the same case. The corresponding results for the case $\gamma>2$ have been obtained recently by Zhang in [21] and [22] respectively.

Let us consider the following initial boundary value problem to (1.1)

$$
\left\{\begin{array}{l}
\rho_{t}+m_{x}=0 \\
m_{t}+\left(\frac{m^{2}}{\rho}+p(\rho)\right)_{x}=\rho \Phi_{x}-\frac{m}{\tau} \\
\Phi_{x x}=\rho-D(x)
\end{array}\right.
$$

with the initial-boundary conditions

$$
\begin{aligned}
& (\rho, m)(x, 0)=\left(\rho_{0}(x), m_{0}(x)\right), \quad 0<x<1 \\
& m(0, t)=0, \quad m(1, t)=0, \quad t \geq 0 \\
& \Phi(0, t)=\Phi_{0}(t), \quad \Phi(1, t)=\Phi_{0}(t), \quad t \geq 0
\end{aligned}
$$

where $\Phi_{0}$ stands for the applied bias and $\rho_{0}(x) \geq 0$.

The solution of the Poission equation $(2.1)_{3}$ with the boundary data $(2.2)_{3}$ is expressed by

$$
\Phi(x, t)=\int_{0}^{1} G(x, t)[\rho(\xi, t)-D(\xi)] d \xi+\Phi_{0}(t)
$$

where $G(x, \xi)$ is the Green's function for this problem and is defined by

$$
G(x, \xi)= \begin{cases}x(\xi-1), & x<\xi \\ \xi(x-1), & x>\xi\end{cases}
$$

Therefore, the system (2.1) can be rewritten as

$$
\left\{\begin{array}{l}
\rho_{t}+m_{x}=0 \\
m_{t}+\left(\frac{m^{2}}{\rho}+p(\rho)\right)_{x}=\rho\left(\int_{0}^{1} G_{x}(\rho-D) d \xi\right)-\frac{m}{\tau}
\end{array}\right.
$$

The global existence of weak solutions to this initial -boundary value problem (2.1) (2.2) was investigated in [20] for the case of $1<\gamma \leq \frac{5}{3}$, but the zero relaxation limit can not be carried out since there is no uniform boundedness results with respect to $\tau$ in [20]. Besides, it was not discussed whether the weak solution satisfies the boundary conditions. 
We discussed the relaxation limit problem for (2.1)(2.2) when $1<\gamma \leq \frac{5}{3}$ and verified that the weak solution satisfies the boundary condition in the sense of trace in [8].

The main steps to establish the result is the follows.

(1) We construct the approximate solutions of the problems $(2.3)(2.2)$ by the modified Godunov scheme and then obtain the uniform boundedness of the approximate solutions with respect to the small momentum relaxation time $\tau$. Namely, partition the interval $(0,1)$ into cells with $\mathrm{j}$-th cell centered at $x_{j}=j h$, where $h$ is the space mesh length, and define the average value of $\rho_{j}^{0}$ and $m_{j}^{0}$ from $x_{j-1}$ to $x_{j}$; consider the corresponding homogenuous system first to solve either the Riemann problem in each interior cell or the mixed problem in the cell related to the boundaries; then modify the value of $m$ according to the terms on the right-hand side of (2.3); take the average values again on each cells from $x_{j-1}$ to $x_{j}$ corresponding to $t=k-0$, where $k$ is the time mesh length, and continue the above procedure. See [8] for the detail. The following theorem is proved in [8].

Theorem 2.1. Suppose that the initial data $\left(\rho_{0}(x), m_{0}(x)\right)$ and the given function $D(x)$ satisfy the conditions:

$$
0 \leq \rho_{0}(x) \leq M_{1}, \rho_{0}(x) \not \equiv 0,\left|m_{0}(x)\right| \leq M_{0} \rho_{0}(x) \text {, and }|D(x)| \leq M_{2}
$$

for some positive constants $M_{0}, M_{1}$, and $M_{2}$. Then the approximate solutions $\left(\rho_{k}, m_{k}\right)$ derived by the modified Godunov scheme are uniformly bounded in the region $\bar{I}_{T} \stackrel{\text { def }}{=}$ $\{(x, t): 0 \leq x \leq 1,0 \leq t \leq T\}$ for any $T>0$; i.e. there is a constant $C(T)$ independent of $k, h$ and $\tau$ such that

$$
0 \leq \rho_{h}(x, t) \leq C,\left|m_{h}(x, t)\right| \leq C \rho_{h}(x, t), \text { for }(x, t) \in \bar{I}_{T}
$$

(2) We prove the $H_{l o c}^{-1}$ compactness of the sequence entropy dissipation measures $\eta\left(v_{h}\right)_{t}+q\left(v_{h}\right)_{x}$ associated with every weak entropy pair $(\eta, q)$ and approximate solutions obtained by the modified Godunov scheme. For this purpose, we first give an energy estimate to the classical mechanical entropy pair by which the desired compactness results in Theorem 2.2 can be obtained with the help of some known estimates obtained by Diperna in [4] and by Ding, Chen and Luo in [3] respectively, see [8] for the detail.

THEOREM 2.2. Assume that the conditions of theorem 2.1 are satisfied and $1<\gamma \leq \frac{5}{3}$. Then $\eta\left(v_{h}\right)_{t}+q\left(v_{h}\right)_{x}$ is compact in $H_{l o c}^{-1}(\Omega)$, for every weak entropy pair $(\eta, q)$ and every open subset $\Omega \subset \bar{I}_{T}$.

Incorporating this $H_{l o c}^{-1}$ compactness and the theorem 2.1 we are able to get the compactness framework theorem needed.

Theorem 2.3. Assume that the approximate solutions $v_{h}(x, t)=$ $\left(\rho_{h}(x, t), m_{h}(x, t)\right)$ satisfy

(i) $0 \leq \rho_{h} \leq c$ and $\left|m_{h}\right|<c \rho_{h}$ a.e. for a positive constant $c$

(ii) The sequence of entropy dissipation measures $\eta\left(v_{h}\right)_{t}+q\left(v_{h}\right)_{x}$ is compact in $H_{l o c}^{-1}(\Omega)$ for every weak entropy pair $(\eta, q)$ and every open bounded set $\Omega \subset \bar{I}_{T}$.

Then for $1<\gamma \leq \frac{5}{3}$, there exists a convergent subsequence, still labeled by $v_{h}$, such that

$$
\left(\rho_{h}(x, t), m_{h}(x, t)\right) \rightarrow(\rho(x, t), m(x, t)) \quad \text { a.e. }
$$


(3) We prove the global existence of weak entropy solutions to (2.3)(2.2), introduce the concept of traces to our weak entropy solutions and verify that the weak solutions satisfy the boundary condition in the sense of traces. To show the global existence of weak entropy solutions we only need to show that the limit obtained in the theorem 2.3 is a weak entropy solution, namely to show the consistency of the scheme and to verify the entropy inequality which can be found in [8]. The concept of traces introduced in [8] is as follows.

Let $v(x, t)=(\rho(x, t), m(x, t))$ be a weak solution to (2.3) obtained by our approach. We introduce the generalized function $A: C_{0}^{1}\left(R^{2}\right) \rightarrow R^{2}$ first: for any $\psi \in$ $C_{0}^{1}\left(R^{2}\right), A(\psi)=-\int_{0}^{T} \int_{0}^{1}\left(v \psi_{t}+f(v) \psi_{x}+F \psi\right) d x d t$ where $f(v)=\left(m, \frac{m^{2}}{\rho}+\frac{\rho^{\gamma}}{\gamma}\right)^{T}, F=$ $\left(0, \rho\left(\int_{0}^{1} G_{x}(\rho-D) d \xi\right)-\frac{m}{\tau}\right)^{T}$. We take $\zeta_{0}, \zeta_{T}, \eta_{0}, \eta_{1} \in C_{0}^{1}(R)$ with

$$
\begin{gathered}
\zeta_{0}(0)=1, \zeta_{0}(T)=0 ; \zeta_{T}(0)=0, \zeta_{T}(T)=1 \\
\eta_{0}(0)=1, \eta_{0}(1)=0 ; \eta_{1}(0)=0, \eta_{1}(1)=1
\end{gathered}
$$

For any $\chi \in C_{0}^{1}(R)$, we define the generalized function

$$
\begin{aligned}
& v^{*}(\cdot, 0)(\chi)=A\left(\chi \cdot \zeta_{0}\right)-\chi(0) A\left(\eta_{0} \cdot \zeta_{0}\right)-\chi(1) A\left(\eta_{1} \cdot \zeta_{0}\right), \\
& v^{*}(\cdot, T)(\chi)=-A\left(\chi \cdot \zeta_{T}\right)+\chi(0) A\left(\eta_{0} \cdot \zeta_{T}\right)+\chi(1) A\left(\eta_{1} \cdot \zeta_{T}\right), \\
& f^{*}(v)(0, \cdot)(\chi)=A\left(\eta_{0} \cdot \chi\right), \\
& f^{*}(v)(1, \cdot)(\chi)=-A\left(\eta_{1} \cdot \chi\right),
\end{aligned}
$$

where $\left(\chi \cdot \zeta_{0}\right)(x, t)=\chi(x) \zeta_{0}(t)$ means the tensor product and the same for others.

By the similar argument as used by Heidrich in [6], we can prove the uniqueness of the defined generalized functions $v^{*}(\cdot, 0)$ and etc. with respect to the choice of functions $\zeta$ or $\eta$ which has the properties in (2.4). In fact, the precise understanding of the generalized functions $v^{*}(\cdot, 0)$ and etc. should be an equivalent class of the forms $v^{*}(\cdot, 0)$ and etc.

Now we can define the trace of $v$ along the segments $(0,1) \times\{0\}$ and $(0,1) \times\{T\}$, and the trace of $f(v)$ along the segments $\{0\} \times(0, T)$ and $\{1\} \times(0, T)$, respectively as $v^{*}(\cdot, 0), v^{*}(\cdot, T), f^{*}(v)(0, \cdot)$ and $f^{*}(v)(1, \cdot)$. Similarly, for any $t \in(0, T)$, we can define $v^{*}(\cdot, t)$ as the trace of $v$ along the segment $(0,1) \times\{t\}$; for any $x \in(0,1)$, define $f^{*}(v)(x, \cdot)$ as the trace of $f(v)$ along the segment $\{x\} \times(0, T)$. It is verified in [8] that the weak solution satisfies the initial-boundary condition in the sense of traces. Namely

ThEOREM 2.4. Let $v_{h}(x, t)=\left(\rho_{h}(x, t), m_{h}(x, t)\right)$ be the approximate solutions of (2.3) constructed and $v(x, t)=(\rho(x, t), m(x, t))$ is the limit function of $v_{h}$ as $h \rightarrow 0$. Then $v(x, t)$ satisfies the initial-boundary conditions in the sense that

$$
\begin{aligned}
& m^{*}(0, t)=m^{*}(1, t)=0, \quad t \in(0, T) \\
& v^{*}(x, 0)=v_{0}(x), \quad x \in(0,1) .
\end{aligned}
$$

(4) We deal with the relaxation limit of entropy weak solutions of $(2.3)(2.2)$ as $\tau \rightarrow 0$. We first state the result in the case of the uniform $L^{\infty}$ estimates, and then describe the result in the case of the uniform $L^{p}$ estimates.

By making the change of the scale $t=\frac{s}{\tau}$, we transform (2.3) into

$$
\begin{aligned}
& \rho_{s}^{\tau}+m_{x}^{\tau}=0 \\
& \left(\tau^{2} m^{\tau}\right)_{s}+\left[\tau^{2} \frac{\left(m^{\tau}\right)^{2}}{\rho^{\tau}}+p\left(\rho^{\tau}\right)\right]_{x}=\rho^{\tau} \int_{0}^{1} G_{x}\left(\rho^{\tau}(\xi, s)-D(\xi)\right) d \xi-m^{\tau}
\end{aligned}
$$


where $\rho^{\tau}(x, s)=\rho\left(x, \frac{s}{\tau}\right), m^{\tau}(x, s)=\frac{1}{\tau} m\left(x, \frac{s}{\tau}\right)$. The following theorems are proved in [8].

ThEOREM 2.5. Let $\left(\rho^{\tau}, m^{\tau}\right)$ be the sequence of solutions to (2.5). Then there exist $\rho \in L^{\infty}$ and $m \in L^{2}$ such that

$$
\begin{aligned}
& \tau \rho^{\tau} \rightarrow \rho \quad \text { a.e. as } \quad \tau \rightarrow 0 \\
& \tau^{\frac{1}{2}} m^{\tau} \rightarrow m \quad \text { as } \quad \tau \rightarrow 0
\end{aligned}
$$

moreover the limit function $(\rho, m)$ satisfies the simplified drift-diffusion equation

$$
\begin{gathered}
\rho_{s}=0 \\
p(\rho)_{x}=0
\end{gathered}
$$

in the sense of distribution. (As far as the case when $\gamma>\frac{5}{3}$ is concerned, a similar result is given by Qiu and Zhang in [19].)

THEOREM 2.6. Let $\left(\rho^{\tau}, m^{\tau}\right)$ be the sequence of solutions to (2.5). Suppose $\gamma=1+\frac{2}{k}$ with $1 \leq k \in N$. Denote $Q_{T}=(0,1) \times(0, T)$. Then there exist $(\bar{\rho}, \bar{m})$ such that

$$
\rho^{\tau} \rightarrow \bar{\rho} \quad \text { as } \tau \rightarrow 0 \quad \text { in } L_{l o c}^{q}\left(Q_{T}\right)
$$

strongly for any $q \in[1, \gamma+1]$,

$$
\begin{gathered}
m^{\tau} \rightarrow \bar{m} \quad \text { as } \tau \rightarrow 0 \quad \text { in } L^{2}\left(Q_{T}\right) \quad \text { weakly, } \\
\frac{\tau^{2}\left(m^{\tau}\right)^{2}}{\rho^{\tau}} \rightarrow 0 \quad \text { as } \tau \rightarrow 0 \quad \text { in } L^{2}\left(Q_{T}\right) \quad \text { strongly. }
\end{gathered}
$$

Moreover, the limit functions $(\bar{\rho}, \bar{m}) \in L^{\infty}\left(0, T ; L^{q}([0,1])\right) \times L^{2}\left(Q_{T}\right)$ for any $q \in$ $(1, \infty)$, satisfy the drift-diffusion equation

$$
\begin{gathered}
\bar{\rho}_{s}+\bar{m}_{x}=0 \\
\bar{m}=\bar{\rho} \int_{0}^{1} G_{x}(x, \xi)(\bar{\rho}(\xi, s)-D(\xi)) d \xi-p(\bar{\rho})_{x}
\end{gathered}
$$

in the sense of distributions.

Turn to the hydrodynamic model in bipolar case next, namely

$$
\left\{\begin{array}{l}
\alpha_{t}+m_{x}=0 \\
m_{t}+\left(\frac{m^{2}}{\alpha}+p(\alpha)\right)_{x}=\alpha \Phi_{x}-\frac{m}{\tau} \\
\beta_{t}+n_{x}=0 \\
n_{t}+\left(\frac{n^{2}}{\beta}+q(\beta)\right)_{x}=-\beta \Phi_{x}-\frac{n}{\tau} \\
\Phi_{x x}=\alpha-\beta-D(x)
\end{array}\right.
$$

where $p(\alpha)=\frac{\alpha^{\gamma_{\alpha}}}{\gamma_{\alpha}}, \gamma_{\alpha}>1 ; q(\beta)=\frac{\beta^{\gamma_{\beta}}}{\gamma_{\beta}}, \gamma_{\beta}>1, \alpha, \beta, m, n$ and $\Phi$ are explained in (1.2). The existence of weak solutions to (2.6) with either initial data or initial boundary 
conditions and the zero relaxation limit to the corresponding drift-diffusion equations (1.4) have been discussed in [18] and [9] respectively. Natalini gave the global existence and zero relaxation limit of weak solutions to the Cauchy problem in [18] for the case $1<\gamma_{\alpha} \leq \frac{5}{3}$ and $1<\gamma_{\beta} \leq \frac{5}{3}$. Hsiao and Zhang solved the following initial-boundary value problem and give the relaxation limit result for the global weak solutions in [9], also proved that the weak solutions satisfy the boundary condition after introducing the concept of the trace to the weak solutions. Consider the initial-boundary value problem

$$
\left\{\begin{array}{l}
(2.6) \\
(\alpha, m, \beta, n)(x, 0)=\left(\alpha_{0}(x), m_{0}(x), \beta_{0}(x), n_{0}(x)\right), 0<x<1, \alpha_{0}(x) \geq 0 \\
\beta_{0}(x) \geq 0 \\
(m, n)(0, t)=(0,0),(m, n)(1, t)=(0,0), \quad t \geq 0 \\
\Phi(0, t)=\Phi_{0}(t), \Phi(1, t)=\Phi_{0}(t), \quad t \geq 0
\end{array}\right.
$$

where $\Phi_{0}$ stands for the applied bias. Using the formular

$$
\Phi(x, t)=\int_{0}^{1} G(x, \xi)[\alpha(\xi, t)-\beta(\xi, t)-D(\xi)] d \xi+\Phi_{0}
$$

where $G(x, \xi)$ is the Green's function as defined before, the system (2.6) can be reduced to

$$
\left\{\begin{array}{l}
\alpha_{t}+m_{x}=0 \\
m_{t}+\left(\frac{m^{2}}{\alpha}+p(\alpha)\right)_{x}=\alpha \int_{0}^{1} G_{x}(\alpha-\beta-D) d \xi-\frac{m}{\tau} \\
\beta_{t}+n_{x}=0 \\
n_{t}+\left(\frac{n^{2}}{\beta}+q(\beta)\right)_{x}=-\beta \int_{0}^{1} G_{x}(\alpha-\beta-D) d \xi-\frac{n}{\tau}
\end{array}\right.
$$

We are able to get a similar result in [9] as obtained for unipolar case in [8]. It would be interesting to discuss other kinds of reasonable boundary conditions and to establish the corresponding results on global existence of weak entropy solutions and to solve the zero relaxation limit problems.

3. Time-asymptotic behavior of smooth solutions. For smooth solutions, we are interested in the question: whether the hydrodynamic model and the driftdiffusion model have the same large-time behavior.

Let us consider the hydrodynamic model in the case of one carrier type, namely (1.1), and assume $\tau=1$ for convenience. Let $E=\Phi_{x}$. The hydrodynamic model takes the form

$$
\left\{\begin{array}{l}
\rho_{t}+m_{x}=0 \\
m_{t}+\left(\frac{m^{2}}{\rho}+p(\rho)\right)_{x}=\rho E-m \\
E_{x}=\rho-D(x)
\end{array}\right.
$$

The corresponding drift-diffusion model is

$$
\left\{\begin{array}{l}
\rho_{t}+\left[\rho E-p(\rho)_{x}\right]_{x}=0 \\
E_{x}=\rho-D(x) \\
m=\rho E-p(\rho)_{x}
\end{array}\right.
$$

for which the stationary solution satisfies

$$
\left\{\begin{array}{l}
{\left[\rho E-p(\rho)_{x}\right]_{x}=0} \\
E_{x}=\rho-D(x) \\
m=\rho E-p(\rho)_{x}
\end{array}\right.
$$


Luo, Natalini and Xin investigated the Cauchy problems to (3.1) and (3.2) in [13]. Under certain conditions on the doping function $D(x)$ and $p(\rho)$, they first proved that there exists a unique smooth solution $(\bar{\rho}, \bar{E})$ to the system (3.3) with the requirement $\bar{\rho}-D(x) \in H^{1}(R)$ and $\bar{E}(-\infty)=\bar{E}$. Furthermore, suppose $O S C_{x \in R} D(x)$ is sufficiently small and the initial $\tilde{\rho}(x)$ for (3.2) is a small perturbation of $\bar{p}(x)$ in certain sense, then there exists a unique global smooth solution to (3.2) which tends to the solution of (3.3) exponentially fast as $t \rightarrow \infty$. At last, suppose $E^{-}=0, O S C_{x \in R} D(x)$ is sufficiently small, the initial data $\rho_{0}(x)$ and $m_{0}(x)$ for $(3.1)$ is a small perturbation of $\bar{\rho}(x)$ and zero respectively in certain sense, then the Cauchy problem to (3.1) has a unique smooth solution which tends to the solution of (3.3) exponentially fast as $t \rightarrow \infty$.

Hsiao and Yang considered the following initial-boundary value problem in [7],

$$
\begin{gathered}
\left\{\begin{array}{l}
(3.1) \\
\rho(x, 0)=\rho_{0}(x)>0, u(x, 0)=u_{0}(x), 0 \leq x \leq 1 \\
(\rho u)(1, t)=0,(\rho u)(0, t)=0, t>0 \\
E(0, t)=0, t>0
\end{array}\right. \\
\left\{\begin{array}{l}
(3.2) \\
\tilde{\rho}(x, 0)=\tilde{\rho}(x)>0,0 \leq x \leq 1 \\
\left(p(\tilde{\rho})_{x}-\tilde{\rho} \tilde{E}\right)(0, t)=0,\left(p(\tilde{\rho})_{x}-\tilde{\rho} \tilde{E}\right)(1, t)=0, t>0 \\
\tilde{E}(0, t)=0, t>0
\end{array}\right.
\end{gathered}
$$

and compared the solutions (3.4) and (3.5) to the solution of (3.6) respectively

$$
\left\{\begin{array}{l}
(3.3) \\
\left(p(\bar{\rho})_{x}-\bar{\rho} \bar{E}\right)(0)=0 \\
\bar{E}(0)=0, \bar{E}(1)=0
\end{array}\right.
$$

Let us assume that $D(x)$ is Lip-continuous for simplicity, and assume that $p(\rho)$ is $C^{3}$-smooth for $\rho>0$ such that

$$
p^{\prime}(\rho)>0, \text { for } \rho>0
$$

It can be proved then that there exists a unique smooth solution $(\bar{\rho}, \bar{E})$ to the problem (3.6). See [7] for the detail.

Turn to the problem (3.5) now. Assume that

$$
p^{\prime \prime \prime}(\rho)<0, \text { for } \rho>0
$$

and $\tilde{\rho}(x)$ is chosen so that

$$
\int_{0}^{1}(\tilde{\rho}(x)-D(x)) d x=0
$$

Let $(\tilde{\rho}, \tilde{E})$ be a solution to $(3.5)$ and $(\bar{\rho}, \bar{E})$ be the unique solution of (3.6). Set $v=\tilde{E}-\bar{E}$. Under the assumptions $(3.7)(3.8)$ and (3.9), we have the following theorem on the large-time behavior of the solution to (3.5).

ThEOREM 3.1. Suppose $O S C_{x \in R} D(x)$ is sufficiently small and $v(\cdot, 0) \in H^{2}$. Assume that the conditions (3.7)-(3.9) hold. Then, there exists a positive number 
$\varepsilon_{0}$ such that if $\|v(x, 0)\|_{H^{2}} \leq \varepsilon_{0}$, then the problem (3.5) has a unique global smooth solution $(\tilde{\rho}, \tilde{E})$ for which it holds that

$$
\begin{gathered}
\|\tilde{E}(\cdot, t)-\bar{E}\|_{H^{2}} \leq \tilde{C} \exp (-\tilde{\alpha} t)\|\tilde{E}(\cdot, 0)-\bar{E}(\cdot)\|_{H^{2}} \\
\|\tilde{\rho}(\cdot, t)-\bar{\rho}(\cdot)\|_{H^{1}} \leq \tilde{C} \exp (-\tilde{\alpha} t)
\end{gathered}
$$

for some positive constants $\tilde{C}$ and $\tilde{\alpha}$.

To prove this theorem, the crucial step is to establish the following a priori estimates.

LEMma 3.2 There exist some positive constants $\delta_{0}>0$ and $\beta_{0}>0$ such that, for any $T>0$, if $\sup _{0 \leq t \leq T}\|v(\cdot, t)\|_{H^{2}} \leq \delta_{0}$ and $\operatorname{osc}_{x \in[0,1]} D(x) \leq \delta_{0}$, then it holds

$$
\|v(\cdot, t)\|_{H^{2}}^{2} \leq \exp \left(-\beta_{0} t\right)\|v(\cdot, 0)\|_{H^{2}}^{2}
$$

for any $t \in[0, T]$. (See [7] for the detail)

We study the problem (3.1) next. Let $(\rho, m, E)$ be a solution to (3.1), and set $\psi=E-\bar{E}$. Assume that $\rho_{0}(x)$ is chosen so that

$$
\int_{0}^{1}\left(\rho_{0}(x)-D(x)\right) d x=0
$$

We have shown the large-time behavior of solution to (3.1) as follows.

Theorem 3.3. Suppose $\operatorname{osc}_{x \in[0,1]} D(x)$ is sufficiently small and $\psi(\cdot, 0) \in$ $H^{3}, m_{0}(\cdot) \in H^{2}$. Assume the conditions (3.7) and (3.10) hold. Then, there exists a positive number $\varepsilon_{1}$ such that if

$$
\|\psi(\cdot, 0)\|_{H^{3}}+\left\|m_{0}(\cdot)\right\|_{H^{2}} \leq \varepsilon_{1}
$$

then the problem (3.1) has a unique smooth solution $(\rho, m, E)$ for which it holds

$$
\|\rho(\cdot, t)-\bar{\rho}(\cdot)\|_{H^{2}}+\|E(\cdot, t)-\bar{E}(\cdot)\|_{H^{3}}+\|m(\cdot, t)\|_{H^{2}} \leq c \exp (-\alpha t)
$$

for some positive constants $c$ and $\alpha$.

To prove this theorem, we have to establish the $a$ priori estimates first.

LEMMA 3.4 There exist some positive constants $\delta_{1}>0$ and $\beta_{1}>0$ such that for any $T>0$, if $\sup _{0 \leq t \leq T}\left(\|\psi(\cdot, t)\|_{H^{3}}+\left\|\psi_{t}(\cdot, t)\right\|_{H^{2}}\right) \leq \delta_{1}$ and $o s c_{x \in[0,1]} D(x) \leq \delta_{1}$, then it holds

$$
\left(\|\psi(\cdot, t)\|_{H^{3}}^{2}+\left\|\psi_{t}(\cdot, t)\right\|_{H^{2}}^{2}\right) \leq c\left(\|\psi(\cdot, 0)\|_{H^{3}}^{2}+\psi_{t}(\cdot, 0) \|_{H^{2}}^{2}\right) \exp (-\beta t)
$$

for any $t \in[0, T]$. See [7] for the detail.

It would be very interesting to establish the corresponding results on smooth solutions for hydrodynamic model and drift-diffusion model in bipolar case.

\section{REFERENCES}

[1] P.DEgond AND P.A.MARKowich, On a one-dimensional steady-state hydrodynamic model for semiconductors, Appl. Math. Letters, 3 (1990), pp. 25-29.

[2] P.Degond and P.A.Markowich, A steady-state potential model for semiconductors, Ann. Mat. Pura. Appl., IV (1993), pp. 87-98. 
[3] X.Ding, G.Q.Chen And P.Luo, Convergence of the fractional step Lax-Friedrichs scheme for isentropic gas dynamics I, Acta Math.Sci., 5 (1985), pp. 415-432.

[4] R.J.Diperna, Convergence of the viscosity method for isentropic gas dynamics, Comm. Math. Phys., 91 (1983), pp. 1-30.

[5] I.M.GAmBA, Stationary transonic solutions of a one-dimensional hydrodynamic model for semiconductors, Comm.P.D.E., 17 (1992), pp. 553-577.

[6] A.Heidrich, Global weak solutions to initial-boundary value problems for the wave equation with large data, Arch. Rat. Mech. Anal., 126 (1994), pp. 333-368.

[7] L.HsiaO AND T.YANG, The asymptotic behavior of solutions of the initial-boundary value problems to the hydrodynamic model and drift-diffusion model in semiconductors, J. Diff. Eqns., 170 (2001), pp. 472-493.

[8] L.HSIAO AND K.J.ZHANG, The relaxation of the hydrodynamic model for semiconductors to the drift-diffusion equation, J. Diff. Eqns., 165 (2000), pp. 315-354.

[9] L.HSIAO AND K.J.ZHANG, The global weak solutions and relaxation limits of the initial-boundary value problem to the bipolar hydrodynamic model for semiconductors, Math. Modeling and Methods in Appl. Science., Vol.10 No.9 (2000), pp. 1333-1361.

[10] J.W.Jerome, Analysis of Charge Transport: A Mathematical Study of Semiconductor Devices, Springer-Verlag, Heidelberg, 1996.

[11] A.JüNGEL, On the existence and uniqueness of transient solutions of a degenerate nonlinear drift-diffusion model for semiconductors, Math. Mod. Meth. Appl. Sci., 4 (1994), pp. 677704.

[12] A.JüNGEL, Qualitative behavior of transient solutions of a degenerate nonlinear drift-diffusion model for semiconductors, Math. Mod. Meth. Appl. Sci., 5 (1995), pp. 497-518.

[13] T.LuO, R.NATAlini AND Z.P.XIN, Large-time behavior of the solutions to a hydrodynamic model for semiconductors, SIAM J.Appl. Math., Vol.59 No.3 (1998).

[14] P.A.MARCATI AND R.NATALINI, Weak solutions to a hydrodynamic model for semiconductors: the Cauchy problem, Proc. Roy. Soc. Edinburgh Sect., A 125 (1995), pp. 115-135.

[15] P.A.Marcati AND NATAlini, Weak solutions to a hydrodynamic model for semiconductors and relaxation to the Drift-Diffusion equation, Arch. Rational Mech. Anal., 129 (1995), pp. 129-145.

[16] P.A.Markowich, On steady-state Euler-Poisson model for semiconductors, Z.Angew. Math. Phys., 62 (1991), pp. 389-407.

[17] P.A.Markowich, C.Ringhofer and C.Schmeiser: Semiconductor Equations, Springer Verlag, Wien, New York (1990).

[18] R.NATALINI, The bipolar hydrodynamic model for semiconductors and the drift-diffusion equation, J. Math. Anal. Appl., 198 (1996), pp. 262-281.

[19] Y.C.QIU AND K.J.ZHANG, On the relaxation limits of the hydrodynamic model for semiconductor devices, preprint.

[20] B.ZHANG, Convergence of the Godunov scheme for a simplified one-dimensional hydrodynamic model for semiconductor devices, Comm. Math. Phys., 157 (1993), pp. 1-22.

[21] K.J.ZHANG, Global weak solutions of the Cauchy problem to a hydrodynamic model for semiconductors, accepted by J. Diff. Eqns.

[22] K.J.ZHANG, Zero relaxation limit of global weak solutions of the Cauchy problem to a hydrodynamic model for semiconductors, preprint. 